\title{
IMPEDANCE AND G-R NOISE OF n-TYPE GOLD-DOPED SILICON UNDER SPACE-CHARGE CONDITIONS
}

\author{
TH. G. M. KLEINPENNING \\ Fysisch Laboratorium, Rijksuniversiteit Utrecht, Nederland
}

Received 18 August 1971

\section{Synopsis}

Impedance and current-noise measurements were made on $\mathbf{n}$-type gold-doped silicon single crystals provided with electron-injecting contacts. The d.c. current is proportional to the applied voltage in the ohmic regime considered here. The capacitance is both frequency and d.c. bias dependent at frequencies below the reciprocal electron transit time. In addition, the capacitance shows a sinusoidal behaviour as a function of frequency under certain conditions. The a.c. resistance, equal to the d.c. resistance at lower frequencies, rapidly decreases at frequencies around the reciprocal freeelectron lifetime and becomes constant at higher frequencies; moreover, the magnitude depends on the d.c. bias. The observed generation-recombination $(\mathrm{g}-\mathrm{r})$ noise is trapping noise associated with a gold-acceptor level at $0.54 \mathrm{eV}$ below the conduction band. The frequency behaviour of the $g-r$ noise spectra depends on d.c. bias voltage at the higher levels where the free-electron transit time becomes smaller than both the free-electron lifetime and the dielectric relaxation time. The free-electron lifetime is found to be about $1 \mu \mathrm{s}$ at room temperature.

1. Introduction. Recently, Zijlstra and Driedonks $\left.{ }^{1-3}\right)$, have derived expressions for the a.c. small-signal impedance and generation-recombination ( $g-r)$ noise of space-charge-limited (SCL) diodes. Their calculations concerned an n-type semiconductor, with one trapping level and fully ionized donors. In addition they assume a planar geometry whereby the contact spacing is small with respect to the transverse dimensions of the diode so that a one-dimensional treatment is allowed. The major approximation made was the neglect of free-carrier diffusion as is usually done in the description of injection devices. In fact, the behaviour of one-carrier, SCL injection currents, is completely dominated by the bulk properties of the diode, provided that the anode and cathode contacts are well separated ${ }^{4}$ ). If the applied d.c. voltage is large compared to $k T / q$ (about $25 \mathrm{mV}$ at room temperature), where $k$ is Boltzmann's constant, $T$ the temperature and $-q$ the electron charge, the current is carried chiefly by drift in the bulk and diffusion can be neglected ${ }^{4}$ ). Shockley and Prim ${ }^{5}$ ) have shown theoretically 
that for most purposes the error owing to neglecting diffusion, is very small compared to the exact solution. Diffusion currents are sizable only in the immediate neighbourhood of the contacts, and neglect of such currents is consistent with omitting a detailed description of the contact area. In the theory the contact properties are accounted for by suitable boundary conditions to the differential equation, which describes the a.c. properties of the bulk. This differential equation will be derived in the next section, starting from the basic equations and using the procedure of Zijlstra and Driedonks ${ }^{1-3}$ ). In addition, by introducing Langevin noise sources ${ }^{8}$ ) we have calculated the trapping and thermal or Nyquist noise.

This paper is primarily concerned with the effects on impedance and current noise of injecting contacts on gold-doped n-type silicon in the ohmic regime.

2. Theory. The one-dimensional rate equation for free electrons in a twolevel model, including fluctuation terms, is:

$$
\frac{\partial n_{1}}{\partial t}=p_{21}-p_{12}+(1 / q) \frac{\partial j_{n}}{\partial x}+H_{1}(x, t)
$$

where $n_{1}$ is the density of free electrons and $j_{n}$ the particle current density. $p_{12}$ is the transition rate per unit volume of electrons between levels 1 and 2 , according to the mass-action law $p_{12}=\alpha_{12} n_{1}\left(n_{2 \mathrm{t}}-n_{2}\right)$, with $\alpha_{12}$ a constant related to the capture probability for an electron transition from level 1 to level 2, and $n_{2 \mathrm{t}}$ the effective density of states of level $2^{7}$ ). In this paper levels 1 and 2 correspond to the conduction band and the gold trapping level, respectively. $H_{1}(x, t)$ is the random Langevin source function associated with the electron trapping and detrapping transitions, with $x$ the longitudinal position coordinate and $t$ the time.

The equation for the total current density $J$, neglecting diffusion, reads:

$$
J=j_{n}+\varepsilon_{\mathbf{r}} \varepsilon_{0} \frac{\partial E}{\partial t}=q \mu n_{1} E+H_{2}(x, t)+\varepsilon_{\mathbf{r}} \varepsilon_{0} \frac{\partial E}{\partial t},
$$

where $J$ is independent of $x$. Here $\varepsilon_{\mathrm{r}} \varepsilon_{0}$ is the permittivity, $E$ the electric field strength, $\mu$ the free-electron drift mobility and $H_{2}(x, t)$ is the random Langevin source function associated with the transport fluctuations according to the thermal-noise theory. Poisson's equation is:

$$
\frac{\partial E}{\partial x}=-\left(q / \varepsilon_{\mathbf{r}} \varepsilon_{0}\right)\left(n_{1}+n_{2}-n_{\mathrm{d}}\right),
$$

where $n_{2}$ is the trapped-electron density and $n_{\mathrm{d}}$ is the density of donor states. 
By considering fluctuations around a steady state, eqs. (1), (2) and (3) can be linearized by putting

$$
n_{i}=n_{i 0}+\Delta n_{i}, \quad J=J_{0}+\Delta J \quad \text { and } \quad E=E_{0}+\Delta E,
$$

where steady-state values are indicated by the subscript 0 and $\Delta n_{1,2}, \Delta J$ and $\Delta E$ are small fluctuations or variations. It should be noted here that $E_{0}$ and $J_{0}$ are directed in the negative $x$ direction. Thence we obtain

$$
\begin{aligned}
\frac{\partial \Delta n_{1}}{\partial t} & =\left[\frac{\partial\left(p_{21}-p_{12}\right)}{\partial n_{1}}\right]_{0} \Delta n_{1} \\
+ & {\left[\frac{\partial\left(p_{21}-p_{12}\right)}{\partial n_{2}}\right]_{0} \Delta n_{2}+(1 / q) \frac{\partial \Delta j_{n}}{\partial x}+H_{1}(x, t) . }
\end{aligned}
$$

Sirce

$$
\frac{\partial \Delta j_{n}}{\partial x}=q \frac{\partial\left(\Delta n_{1}+\Delta n_{2}\right)}{\partial t}, \quad \frac{1}{\tau_{1}}=\left[\frac{\partial\left(p_{12}-p_{21}\right)}{\partial n_{1}}\right]_{0}
$$

and

$$
\frac{1}{\tau_{2}}=\left[\frac{\partial\left(p_{21}-p_{12}\right)}{\partial n_{2}}\right]_{0}
$$

we find:

$$
\frac{\partial \Delta n_{2}}{\partial t}=\frac{\Delta n_{1}}{\tau_{1}}-\frac{\Delta n_{2}}{\tau_{2}}-H_{1}(x, t) .
$$

Here $\tau_{1}$ and $\tau_{2}$ are the free- and trapped-electron lifetimes, respectively.

In addition we have:

$$
\begin{aligned}
& \Delta J=q \mu n_{10} \Delta E+q \mu E_{0} \Delta n_{1}+\varepsilon_{\mathrm{r}} \varepsilon_{0} \frac{\partial \Delta E}{\partial t}+H_{2}(x, t), \\
& \frac{\partial \Delta E}{\partial x}=-\left(\frac{q}{\varepsilon_{\mathrm{r}} \varepsilon_{0}}\right)\left(\Delta n_{1}+\Delta n_{2}\right) .
\end{aligned}
$$

By eliminating $\Delta n_{1}$ and $\Delta n_{2}$ in eq. (4a) with the help of eqs. (4b' and $(4 c)$, we find a differential equation for $\Delta E$. By making a Fourier transform from time to frequency of this equation for $\Delta E$, we find, using some algebra $\left.^{1-3}\right)$ :

$$
\frac{\mathrm{d} e(\omega, x)}{\mathrm{d} x}+a(\omega) e(\omega, x)=c(\omega, x),
$$

where

$$
a(\omega)=\left[t_{n \tau_{2}}\left(1+\mathrm{i} \omega \tau_{\Omega}\right)(1+\mathrm{i} \omega \tau)\right] /\left[\tau_{\Omega} \tau\left(1+\mathrm{i} \omega \tau_{2}\right) L\right]
$$


and

$$
\begin{gathered}
c(\omega, x)=\left[j(\omega)-h_{2}(\omega, x)\right] \rho a(\omega) /\left(1+\mathrm{i} \omega \tau_{\Omega}\right) \\
+\left(q / \varepsilon_{\mathrm{r}} \varepsilon_{0}\right) \tau_{2} h_{1}(\omega, x) /\left(1+\mathrm{i} \omega \tau_{2}\right) .
\end{gathered}
$$

Here $\omega=2 \pi f$ is the angular frequency; $t_{n}=-L /\left(\mu E_{0}\right)$ is the electron transit time provided that $\mathrm{d} E_{0} / \mathrm{d} x=0 ; L$ is the cathode-anode spacing in a planar geometry; $\tau=\tau_{1} \tau_{2} /\left(\tau_{1}+\tau_{2}\right)$ the trapping relaxation time of free electrons; $\tau_{\Omega}$ the dielectric relaxation time [equal to $\varepsilon_{\mathrm{r}} \varepsilon_{0} \rho$ where $\rho=1$ / $\left(q \mu n_{1}\right)$ is the resistivity $\rfloor . e(\omega, x), j(\omega), h_{1}(\omega, x)$ and $h_{2}(\omega, x)$ are the Fourier transforms of $E, J, H_{1}$ and $H_{2}$, respectively.

By putting $h_{1,2}(\omega, x) \equiv 0$ in eq. (5) one finds a unique solution for $e(\omega, x)$ in terms of $j(\omega)$, provided that a boundary condition is given. The a.c. voltage $v(\omega)$ across the diode is given by $v(\omega)=-\int_{0}^{L} e(\omega, x) \mathrm{d} x$. Therefore the a.c. impedance $Z(\omega)$ can be found according to $Z(\omega)=-v(\omega)$ / $[j(\omega) A]$, where $A$ is the cross-sectional area of the sample. On the other hand, by substitution of $j(\omega) \equiv 0$ in eq. (5) the spectral density of the a.c. open-circuit voltage fluctuations, $S_{\Delta V}$, can be calculated. Thence the a.c. short-circuit current fluctuations, $S_{\Delta I}$, may be obtained from $S_{\Delta I}=S_{\Delta V} /$ $|Z|^{2}$.

In order to check this theory we measured the a.c. impedance and the generation-recombination noise of high-ohmic n-type gold-doped silicon samples, provided with either electron space-charge injecting contacts or non-injecting contacts. We prefer gold-doped silicon on account of its properties ${ }^{6,7}$ ) and since it is easily obtainable.

With injecting contacts one has a large reservoir of electrons in the immediate vicinity of the injecting $\mathrm{n}^{+}$contact, accordingly we put $e(x, \omega)=0$ at $\left.x=0^{4}\right)$. We have non-injecting contacts if the total charge of the sample, $N_{1}+N_{2}-N_{\mathrm{d}}$, is zero, so that $\int_{0}^{L}(\partial E / \partial x) \mathrm{d} x=0$ where $N_{1}$ and $N_{2}$ are the total numbers of free and trapped electrons in the sample, respectively, and $N_{\mathrm{d}}$ is the total number of donor states in the sample [cf. e.q. (3)] and hence $\int_{0}^{L}[\mathrm{~d} e(\omega, x) / \mathrm{d} x]=0$. Note that the cathode contact is localized at $x=0$ and the anode contact at $x=L$.

For our measurements the conditions

$$
\tau_{2} \gg \tau \quad \text { and } \quad\left(t_{n} \tau_{2}\right) /\left(\tau_{\Omega} \tau\right)=t_{n}^{\prime} / \tau_{\Omega}=a(0) L \gg 1
$$

were met. Here $t_{n}^{\prime}=t_{n}\left(\tau_{2} / \tau\right)$ is the effective transit time of an electron, because $\tau / \tau_{2}=\tau_{1} /\left(\tau_{1}+\tau_{2}\right)$ is the fraction of time in which the electron will be free. Note that by eliminating $\Delta n_{2}$ (or $\Delta n_{1}$ ) and $\Delta E$ in eqs. (4a)-(4c), we find a macroscopic differential equation for $\Delta n_{1}$ (or $\Delta n_{2}$ ), by putting $H_{1}(x, t)=H_{2}(x, t) \equiv 0$. By making a Fourier transform from time to frequency of this equation for $\Delta n_{i}(i=1,2)$, we find, using $\mathrm{d} j(\omega) / \mathrm{d} x=0$, $\mathrm{d} \tilde{n}_{i} / \mathrm{d} x+a(\omega) \tilde{n}_{i}=0$, where $\tilde{n}_{i}$ is the Fourier transform of $\Delta n_{i}$. Consequently we have $\mathrm{d}\left(\tilde{n}_{1}+\tilde{n}_{2}\right) / \mathrm{d} x+a(\omega)\left(\tilde{n}_{1}+\tilde{n}_{2}\right)=0$, hence it follows that 
$\operatorname{Re}[a(\omega)]$ is the reciprocal penetration depth of the injected a.c. spacecharge at the injecting contact. The condition $a(0) L \gg 1$ implies that most of the injected d.c. charge will be trapped in the vicinity of the cathode contact. This means also that the injected d.c. charge is kept so small, that the densities of free electrons and empty traps are not affected in the larger part of the samples. As a consequence the d.c. $I-V$ characteristic is ohmic, since the applied d.c. voltage was chosen below the limit where Child's law holds $\left.{ }^{4}\right)$ (i.e. the inequality $t_{n}^{\prime} \gg \tau_{\Omega}$ prevails). Another consequence of the conditions $t_{n}^{\prime} \gg \tau_{\Omega}$ and $\tau_{2} \gg \tau$ are, that $\tau, \tau_{2}, \tau_{\Omega}, E_{0}$ and $a(\omega)$ are independent of the position coordinate $x$ in the larger part of the sample. Once again we note that the equation $\mathrm{d} \tilde{n}_{i} / \mathrm{d} x+a(\omega) \tilde{n}_{i}=0$ is not valid in the immediate vicinity of the cathode contact, since there the diffusion currents are sizable and in addition the quantity $a(\omega)$ depends on the position coordinate in the contact area.

We obtain in the case of non-injecting contacts by integration of eq. (5).

$$
v(\omega)=-[1 / a(\omega)] \int_{0}^{L} c(\omega, x) \mathrm{d} x
$$

where $v(\omega)$ is the Fourier transform of $V$ and $V$ is the voltage over the sample.

By putting $h_{1,2}(\omega, x) \equiv 0$ and $\tau_{\Omega}=R_{0} C_{0}$, we find

$$
Z=R_{0} /\left(1+\mathrm{i} \omega R_{0} C_{0}\right) \text {, }
$$

where $R_{0}$ and $C_{0}$ are the d.c. resistance and the geometric capacitance of the sample, respectively $\left(C_{0}=\varepsilon_{\mathrm{r}} \varepsilon_{0} A / L\right)$.

$S_{\Delta I}$ can be obtained in the way described by Zijlstra and Driedonks ${ }^{1-3}$ ). Thereby it will be assumed that the spectral cross densities $S_{H_{1}}$ and $S_{H_{2}}$ can be written as ${ }^{1-3,8)}$ :

$$
S_{H_{1}}\left(x_{1}, x_{2}, \omega\right)=\left(4 n_{10} / A \tau_{1}\right) \delta\left(x_{1}-x_{2}\right) \approx\left(4 n_{10} / A \tau\right) \delta\left(x_{1}-x_{2}\right)
$$

and

$$
S_{H_{2}}\left(x_{1}, x_{2}, \omega\right)=\left(4 k T q \mu n_{10} / A\right) \delta\left(x_{1}-x_{2}\right),
$$

respectively, where $\delta$ is the Dirac delta function. In addition we assume that $H_{1}(x, t)$ and $H_{2}(x, t)$ fluctuate independently. Hence we find:

$$
S_{\Delta I}=\left(4 \tau / N_{1}\right) I^{2} /\left(1+\omega^{2} \tau^{2}\right)+4 k T / R_{0},
$$

where $I$ is the d.c. current flow through the sample.

In the case of electron-injecting contacts we obtain $\left.{ }^{1,2}\right)$ :

$$
v(\omega)=-[1 / a(\omega)] \int_{0}^{L} c(\omega, x)\{1-\exp [a(\omega)(x-L)]\} \mathrm{d} x,
$$

so that we find ${ }^{1,2}$ )

$$
Z=\left[R_{0} /\left(1+i \omega R_{0} C_{0}\right)\right] g(a L)
$$


and

$$
S_{\Delta I}=\left[\left(4 \tau / N_{1}\right) I^{2} /\left(1+\omega^{2} \tau^{2}\right)+4 k T / R_{0}\right] f(a L) /|g(a L)|^{2},
$$

where

$$
g(y)=1-1 / y+\exp (-y) / y
$$

and

$$
f(y)=1-\left[\exp \left(-y-y^{*}\right)-1\right] /\left(y+y^{*}\right)+2 \operatorname{Re}\{[\exp (-y)-1] / y\} .
$$

The asterisks denote the complex conjugate quantities.

Note that in eqs. (8) and (11) both the g-r noise and the thermal or Nyquist noise are represented. Eqs. (10) and (11) have been derived already by Driedonks and Zijlstra ${ }^{1-2}$. They have omitted, however, the contributions of the thermal noise. The effect of the functions $g(a L)$ and $f(a L)$ on the impedance and noise are considered in detail in sections 4.3 and 4.4 .

Several remarks should be made.

First, the origin of thermal noise is the thermal motion of the free electrons. It seems inconsistent that in our case the macroscopic representative of these processes, i.e. the diffusion term, in the current equation was omitted. One can prove, however, that this is correct, provided that the dielectric diffusion length, $\left(D_{\tau_{\Omega}}\right)^{\frac{1}{2}}$, where $D$ is the diffusion coefficient of free electrons, may be neglected with respect to the contact spacing ${ }^{9}$ ). In our case this condition obviously holds.

Second, the spectral cross density, $S_{H_{2}}\left(x_{1}, x_{2}, \omega\right)$, only holds at frequencies below the reciprocal collision time of free electrons $\left(\approx 10^{13} \mathrm{~Hz}\right)$.

Third, in the limiting case, where $\omega=0$, one can prove that eq. (10) can be approximated by $Z=R_{0}\{1-1 /[a(0) L]\}$. The small deviation from the value $R_{0}$ is a result of the penetration depth of the injected d.c. spacecharge.

Fourth, the spectral density of the a.c. short-circuit current fluctuations as presented in eqs. (8) and (11) can be calculated also directly from eq. (5) by substitution of $v(\omega) \equiv 0$ instead of $j(\omega) \equiv 0$, although the algebra is more complicated.

Fifth, the assumption that the free-electron mobility is independent of the electric field strength will be used in all calculations.

3. Experiments. 3.1. Preparation of the samples. Investigations were made on $\mathrm{n}$-type gold-doped silicon single crystals as obtained from Metallurgie Hoboken (Belgium). The crystals were float-zoned grown in the (111) direction with an etch pit density of about $10^{4} \mathrm{~cm}^{-2}$ and had gold concentrations of about $10^{15} \mathrm{~cm}^{-3}$. Samples were cut by us in the desired form with a diamond saw, polished with carborundum and etched in $\mathrm{HNO}_{3}-\mathrm{HF}$. Before applying contacts the samples were given a $30 \mathrm{~s}$ dip in 
$\mathrm{HF}$ and then ultrasonically cleaned in distilled water. Non-injecting contacts (nic) were applied to the samples by electroless nickel plating ${ }^{10}$ ). Electron-injecting contacts (eic) were obtained by annealing the samples for three hours at temperatures of about $850^{\circ} \mathrm{C}$ in evacuated and sealed quartz tubes ${ }^{11}$ ), containing small amounts of antimony. Good electroninjecting contacts resulted in this case, as can be checked by impedance measurements under d.c. bias voltage.

Annealing the samples at higher temperatures $\left(1000-1200^{\circ} \mathrm{C}\right)$ resulted in a decrease in the resistivity $\rho$ from about $3 \times 10^{4}$ to $50 \Omega \mathrm{cm}$; the samples remain $n$-type. This decrease may be due either to in-diffusion of antimony or to out-diffusion of gold and subsequent alloying with the antimony layer attached to the sample surface. On account of the fact that the decrease in the resistivity is independent of annealing temperatures between 1000 and $1200^{\circ} \mathrm{C}$ and taking into consideration that antimony has a much smaller diffusion coefficient in silicon than gold at these temperatures ${ }^{11}$ ) (about $10^{-13}$ and $10^{-7} \mathrm{~cm}^{2} / \mathrm{s}$, respectively), we assume that the decrease in the resistivity is caused by out-diffusion of gold. On the other hand, it appears that the resistivity of n-type silicon changes from 50 to $3 \times 10^{4} \Omega \mathrm{cm}$ after addition of a gold concentration of about $10^{15} \mathrm{~cm}^{-312}$, which is of a similar order of magnitude as the original gold concentrations in our samples.

The heat treatment at $850^{\circ} \mathrm{C}$ and addition of antimony was found to give rise to $n^{4}$-contacts, most probably brought about by out-diffusion of gold atoms which normally act as electron traps $\left.{ }^{6}\right)$. Such an $n^{+}$-contact is able to inject clcctrons $\left.{ }^{4}\right)$. The thin $n^{+}$-layer on the sides of the samples, apart from the contacts, were removed by polishing with carborundum. Copper wires were attached to the contacts with the help of silverpaste (Argentol 70).

We indeed found that the problem of fabricating a suitable injecting contact is more often than not a difficult one and that a significant portion of our research program is spent in solving the contact problem.

3.2. Experimental methods. The samples under investigation were mounted on a Peltier battery (Philips PT 47/5) in order to obtain sample temperatures between $250 \mathrm{~K}$ and $300 \mathrm{~K}$, and were masked from the open air by means of vacuum degrease. The temperature was measured with the help of a copper-constantan thermocouple.

Measurements of the a.c. small-signal impedance, as function of frequency and d.c. bias voltage, were performed in two frequency ranges. At higher frequencies $(30 \mathrm{kHz}-10 \mathrm{MHz}$ ) we employed a radio-frequency bridge (Hatfield Instruments Ltd, type LE 300/A1), which could be used under d.c. bias conditions of at most $100 \mathrm{~V}$, without affecting the balance equations of the bridge. This balancing of the bridge is done by means of two dials, calibrated in terms of an equivalent parallel $R_{\mathrm{p}} C_{\mathrm{p}}$ circuit. At frequencies 
below $30 \mathrm{kHz}$ the impedance measurements were made with the help of a lock-in amplifier/phase detector (PAR, model 121). Here the equivalent $R_{p}$ and $C_{\mathrm{p}}$ values can be calculated from the measured values of the phase shift between the applied a.c. voltage and the a.c. current flow through the sample, and the absolute value of the impedance $|Z|$. All reported values of $C_{\mathrm{p}}$ are corrected for parasitic capacitances of about $2.5 \mathrm{pF}$.

The noise measurements were performed with the help of the already mentioned lock-in amplifier at frequencies up to $150 \mathrm{kHz}$, and with a selective microvoltmeter (Rohde and Schwarz, type USVH, BN 1521/2) at the higher frequencies. Calibration of the noise spectra was done by means of a white-noise generator (Quan Tech, model 420). The spectral noise density is expressed in terms of the spectral density $S_{\Delta I}$ (in $A^{2}$ S) of the a.c. short-circuit current fluctuations.

3.3. Results. The results presented in this paper were obtained with a nic-sample with a contact spacing $L=(1.5 \pm 0.05) \mathrm{mm}$ and a cross-sectional area $A=(12 \pm 0.5) \mathrm{mm}^{2}$ and an eic-sample with dimensions

$$
L=(1.0 \pm 0.05) \mathrm{mm} \quad \text { and } \quad A=(13 \pm 0.5) \mathrm{mm}^{2} \text {, }
$$

respectively. Fig. 1 sliows the lemperature dependence of both the resistivity $\rho$, and the relaxation time $\tau$ obtained both from the $g-r$ noise and as found from the capacitance $C_{p}$ in the case of the eic-sample. Fig. 2 shows $R_{\mathrm{p}}$ and $C_{\mathrm{p}}$ of the eic-sample as function of frequency, whereby a d.c. bias voltage of $100 \mathrm{~V}$ was applied to the sample. The measurements were carried out for various values of the d.c. resistance $R_{\boldsymbol{0}}$, obtained by varying the sample temperature. Fig. 3 shows the dependence of $R_{\mathrm{p}}$ and $C_{\mathrm{p}}$ on the ap-

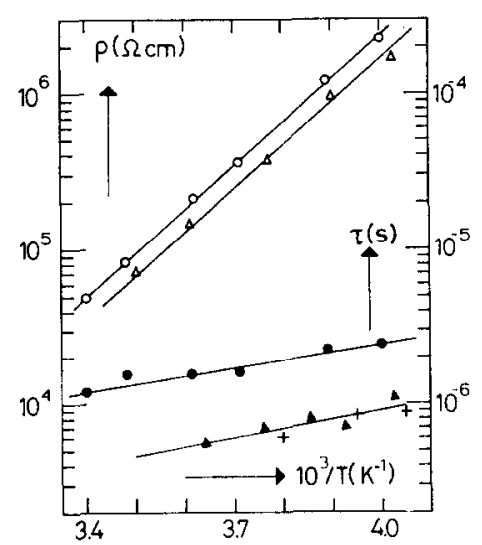

Fig. 1. Temperature dependence of the resistivity $\rho(0, \Delta)$ and the relaxation time $\tau$ obtained both from noise spectra $(\boldsymbol{\theta}, \Delta)$ and from impedance measurements $(+)$. Results obtained with electron-injecting contacts are indicated by $\Delta, \mathbf{\Delta},+$ and with non-injecting contacts by 0 , $\bullet$. The slope of $\rho$ yields $0.55 \mathrm{eV}$. 


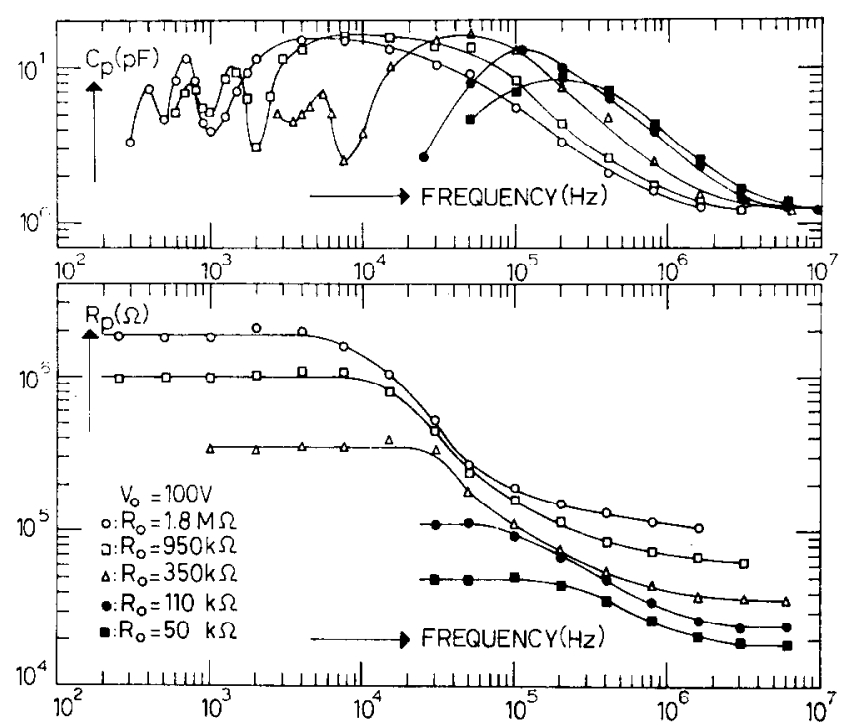

Fig. 2. Equivalent parallel resistance $R_{\mathrm{p}}$ and capacitance $C_{\mathrm{p}}$ as a function of frequency for the sample provided with electron-injecting contacts. Various values of d.c. resistance $R_{0}$ are obtained by varying the sample temperature. The applied d.c. bias voltage $V_{0}$ is $100 \mathrm{~V}$ and the geometric capacitance $C_{0}$ is $1.3 \mathrm{pF}$.

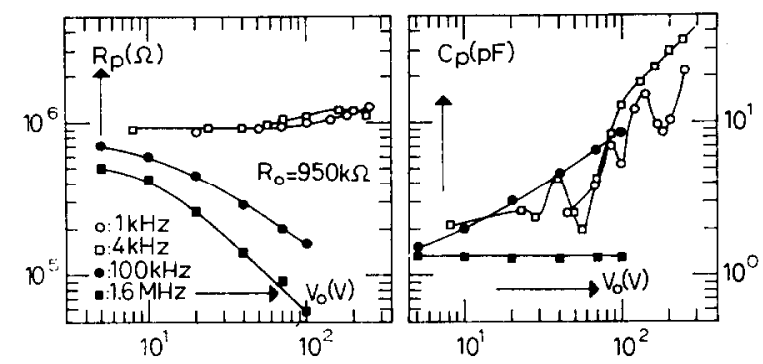

Fig. 3. Equivalent parallel resistance $R_{\mathrm{p}}$ and capacitance $C_{\mathrm{p}}$ as a function of the applied d.c. bias voltage $V_{0}$ for the sample provided with electron-injecting contacts, at several fixed frequencies. The d.c. resistance $R_{0}$ is $950 \mathrm{k} \Omega$ and the geometric capacitance $C_{0}$ is $1.3 \mathrm{pF}$.

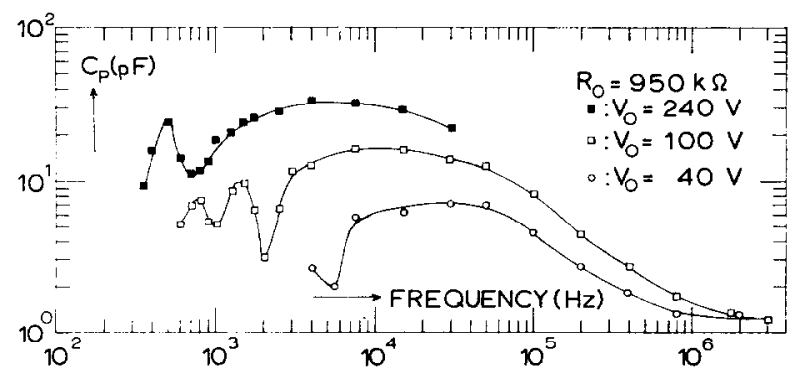

Fig. 4. Equivalent parallel capacitance $C_{\mathrm{p}}$ as a function of frequency for the sample provided with electron-injecting contacts, at several fixed d.c. bias voltages $V_{0}$. The d.c. resistance $R_{0}$ is $950 \mathrm{k} \Omega$ and the geometric capacitance $C_{0}$ is $1.3 \mathrm{pF}$. 


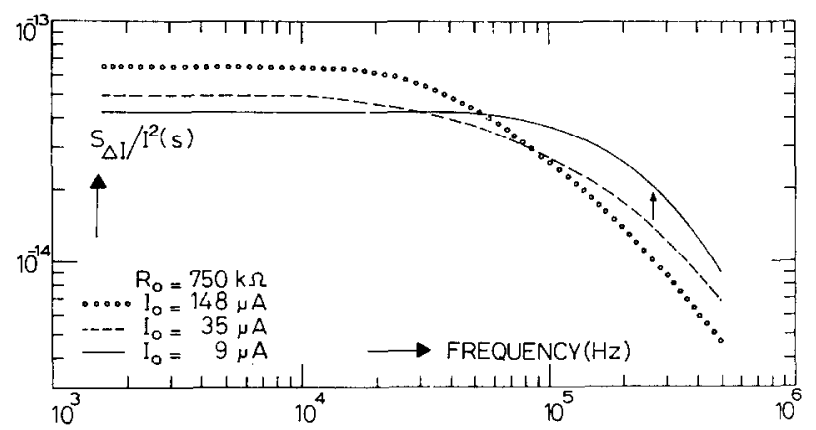

Fig. 5. Typical g-r noise spectra obtained with the sample provided with electroninjecting contacts. At the higher injection levels $(35 \mu \mathrm{A}$ and $148 \mu \mathrm{A})$ the deviation from the ideal shape of the $\mathrm{g}-\mathrm{r}$ noise, namely $\sim 1 /\left(1+\omega^{2} \tau^{2}\right)$ and obtained for $I=9 \mu \mathrm{A}$, is clear. The arrow indicates the cross-over frequency corresponding to the relaxation time $\tau$.

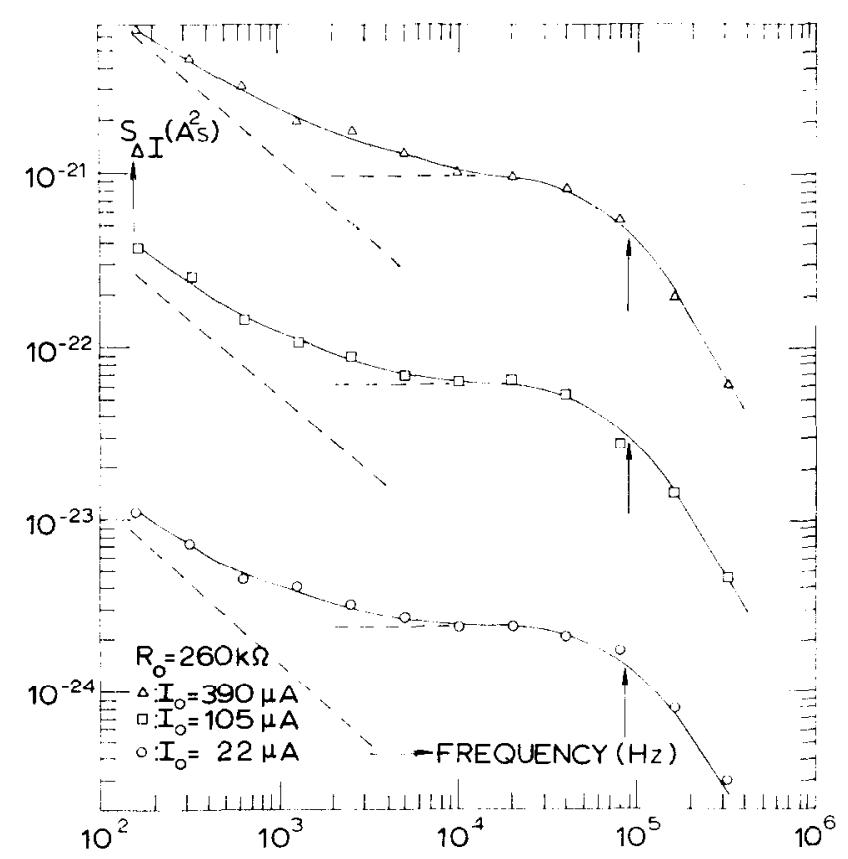

Fig. 6. Typical noise spectra obtained with the sample provided with non-injecting contacts. The spectra can be decomposed in a flicker-noise component and a $\mathrm{g}-\mathrm{r}$ noise component (see dashed lines). The arrows indicate cross-over frequencies corresponding to the relaxation time $\tau$. 
plied d.c. bias voltage at several fixed frequencies. It should be noted that the local maxima and minima of $C_{\mathrm{p}}$ shown in fig. 3 , can be observed directly from the PAR phase detector by varying the d.c. bias voltage. The dependence of $C_{\mathrm{p}}$ on frequency at several fixed d.c. bias voltages is shown in fig. 4 , here the sample d.c. resistance amounts to $950 \mathrm{k} \Omega$.

Concerning the nic-sample, we found that $R_{\mathrm{p}}=R_{0}$ and $C_{\mathrm{p}}=C_{0}$, independent of both the frequency up to $10 \mathrm{MHz}$ and d.c. bias voltages up to $100 \mathrm{~V}$.

Some noise spectra, of the eic-sample and the nic-sample corrected for noise contributions at zero d.c. current, are plotted in fig. 5 and fig. 6 , respectively. The spectra show clearly g-r noise. The increase in the spectral noise density at lower frequencies, shown in fig. 6 , may be attributed to flicker noise. In fig. 5 we have plotted $S_{\Delta I} / I^{2}$ instead of $S_{\Delta I}$. Here the deviation from the ideal frequency behaviour $\left[\right.$ i.e. $\sim 1 /\left(1+\omega^{2} \tau^{2}\right)$ and obtained for $I=9 \mu \mathrm{A}]$ becomes clear at higher injection levels, i.e. higher d.c. currents. At frequencies below $1 \mathrm{kHz}$ the spectral density increases owing to flicker noise. For clarity the data points arc omitted in fig. 5. It should be noted that the relaxation time $\tau$ is derived from noise spectra at lower d.c. currents in case of the eic-samples.

4. Interpretation. 4.1. Sample resistivity. It is well known that gold in silicon gives rise to a donor level at $0.35 \mathrm{eV}$ above the valence band and an acceptor level at $0.54 \mathrm{eV}$ below the conduction band $\left.{ }^{6}\right)$. The band gap is $1.13 \mathrm{eV}$ at room temperature ${ }^{13}$ ). Our samples had been compensated with shallow donors (shallow with respect to the conduction band), for instance phosphorus or antimony ${ }^{13}$ ). As a result the Fermi level will be situated close above the gold-acceptor level (about several $k T$ at room temperature). Since in the temperature range $250 \mathrm{~K}-300 \mathrm{~K}$, the density of empty acceptor states largely exceeds the density of free electrons in the conduction band, it follows that $n_{1}=n_{\mathrm{c}} n_{\mathrm{d}} /\left(n_{\mathrm{t}}-n_{\mathrm{d}}\right) \exp \left(E_{\mathrm{t}} / k T\right)$, where $E_{\mathrm{t}}<0 . E_{\mathrm{t}}$ is the energy of the trapping level with respect to the conduction band, $n_{\mathbf{c}}$ is the effective density of states in the conduction band and $n_{\mathrm{t}}$ is the trap density. Since the temperature dependence of the drift mobility $\mu$ and the density of states in the conduction band $n_{\mathrm{c}}$ nearly cancel out each other $\left.{ }^{13}\right)$, the resistivity can be described by $\rho=1 /\left(n_{1} \mu q\right)=\rho_{0} \exp \left(-E_{\mathrm{t}} / k T\right)$, which corresponds with the activation energy of the experimental data $(0.55 \pm 0.02)$ $\mathrm{eV}$ (cf. fig. 1). For both samples a difference in the resistivity is found, which may be due to inhomogeneities in the boule as grown. We did find that the resistivity at room temperature varies between $2.5 \times 10^{4}$ and $5 \times 10^{4} \Omega \mathrm{cm}$ for different samples cut from the boule. Also a difference in the relaxation time $\tau$ was found for these samples.

Both samples meet Ohm's law up to $1500 \mathrm{~V} / \mathrm{cm}$, whereas at higher fields there arises a small deviation from Ohm's law since the electron drift mo- 
bility becomes field dependent and decreases with increasing field strength ${ }^{14}$ ). Both this effect of field-dependent mobility and space-charge injection cause the increase in the a.c. resistance $R_{\mathrm{p}}$ with increasing d.c. bias voltage at lower frequencies shown in fig. 3 (see also section 4.3).

4.2. Impedance and noise of the nic-sample. From the theory it follows that $R_{\mathrm{p}}=R_{0}$ and $C_{\mathrm{p}}=C_{0}$ [see eq. (7)]. Indeed we found that our impedance measurements are in agreement with the theory; this means that $R_{\mathrm{p}}$ and $C_{\mathrm{p}}$ are independent of both frequency and d.c. bias voltage.

Concerning the $g-r$ noise, the theory predicts the relation represented in eq. (8). The free-electron fluctuations result from electron transitions between the conduction band and the gold-acceptor level, while the golddonor level will be frozen-in and the shallow-donor level is fully ionized.

The noise spectra found can be decomposed in a flicker noise component and an ideal g-r component i.e. $S_{\Delta I} \sim 1 /\left(1+\omega^{2} \tau^{2}\right)$ (see fig. 6). The relaxation time $\tau$ weakly depends on sample temperature (see fig. 1). In our case we find with the help of eq. (4a), the definition of $p_{i j}$ and the inequality $n_{1} \ll n_{\mathrm{c}}: 1 / \tau_{1}=\alpha_{12}\left(n_{\mathrm{t}}-n_{2}\right)_{0}$ and $1 / \tau_{2}=\alpha_{12} n_{10}+\alpha_{21} n_{\mathrm{c}}$. Since for the steady state $\left(p_{12}\right)_{0}=\left(p_{21}\right)_{0}$, we have $1 / \tau_{2}=\alpha_{12}\left(n_{1} / n_{2}\right)_{0} n_{\mathrm{t}}$. Hence the relaxation time $\tau$ is given by:

$$
\begin{aligned}
1 / \tau & =1 / \tau_{1}+1 / \tau_{2}=\alpha_{12}\left\{\left[\left(n_{1}+n_{2}\right) / n_{2}\right] n_{\mathrm{t}}-n_{2}\right\}_{0} \\
& \approx \alpha_{12}\left(n_{\mathrm{t}}-n_{2}\right)_{0}=v_{n} \gamma\left(n_{\mathrm{t}}-n_{2}\right)_{0}
\end{aligned}
$$

where $\left.\alpha_{12}=v_{n} \gamma^{7}\right) ; v_{n}$ is the thermal velocity of the electrons and $\gamma$ is the capture cross section of the gold acceptor for electrons. The temperature dependence of $\tau$ may be ascribed to the temperature dependence of the capture cross section $\gamma^{15}$ ), since the temperature dependence of both $v_{n}$ and the density of empty traps $\left(n_{1}-n_{2}\right)$ will be small.

From Fermi-Dirac statistics it follows that

$$
n_{\mathrm{t}}-n_{2}=n_{\mathrm{t}} /\left\{1+\exp \left[\left(E_{\mathbf{F}}-E_{\mathrm{t}}\right) / k T\right]\right\},
$$

where $E_{\mathbf{F}}=k T \ln \left(n_{1} / n_{\mathbf{c}}\right)$ represents the position of the Fermi level with respect to the bottom of the conduction band, so we find that $n_{\mathbf{t}}-n_{2} \approx$ $\approx 4 \times 10^{13} \mathrm{~cm}^{-3}$ at room temperature, thereby using $\left.{ }^{13}\right) \mu=1.3 \times 10^{3} \mathrm{~cm}^{2}$ l Vs, $n_{\mathrm{c}}=2.8 \times 10^{19} \mathrm{~cm}^{-3}$ and $n_{\mathrm{t}}=10^{15} \mathrm{~cm}^{-3}$. Hence it appears that the capture cross section $\gamma$ is about $10^{-15} \mathrm{~cm}^{2}$ at room temperature.

With respect to the magnitude of the plateau level of the $g-r$ noise, we may conclude that, but for a factor two, good agreement exists between experimental results and the theory of section 2 .

Moreover, the experimental results are in agreement with those obtained by other authors 6,7 ), even at higher voltages, whereby the transit time of electrons becomes smaller than the dielectric relaxation time.

4.3. Impedance of the eic-sample. The results of the measurements 
on the eic-sample strongly differ from the results of the nic-sample. It was found that the data obtained with the eic-sample can be properly described with the help of the theory presented in section 2 .

Equating the a.c. impedance $Z$ from eq. (10) to $R_{\mathrm{p}} /\left(1+\mathrm{i} \omega R_{\mathrm{p}} C_{\mathrm{p}}\right)$, i.e. considering the sample as an equivalent parallel RC circuit, we can express $R_{\mathrm{p}}$ and $C_{\mathrm{p}}$ in terms of $R_{\mathbf{0}}, C_{0}$ and $a L$

$$
R_{\mathrm{p}}=R_{0}\left(r^{2}+s^{2}\right) /\left(r+s \omega \tau_{\Omega}\right) \quad \text { and } \quad C_{\mathrm{p}}=C_{0}[r-s /(\omega \tau \Omega)] /\left(r^{2}+s^{2}\right),
$$

where

$$
\begin{aligned}
r= & \operatorname{Re}[g(a L)]=\left[X^{2}+Y^{2}-X+X \cos Y \exp (-X)\right. \\
& -Y \sin Y \exp (-X)] /\left(X^{2}+Y^{2}\right), \\
s= & \operatorname{Im}[g(a L)]=[Y-X \sin Y \exp (-X) \\
& -Y \cos Y \exp (-X)] /\left(X^{2}+Y^{2}\right),
\end{aligned}
$$

and

$$
\begin{aligned}
X= & \operatorname{Re}(a L) \\
& =\left\{\left(t_{n} \tau_{2}\right) /\left(\tau_{\Omega} \tau\right)\left[1+\omega^{2}\left(\tau_{2} \tau_{\Omega}+\tau_{2} \tau-\tau \tau_{\Omega}\right)\right]\right\} /\left(1+\omega^{2} \tau_{2}^{2}\right), \\
Y= & \operatorname{Im}(a L) \\
& =\left[\left(t_{n} \tau_{2}\right) /\left(\tau_{\Omega} \tau\right)\left(\omega^{3} \tau_{\tau_{\Omega}} \tau_{2}-\omega\left(\tau_{2}-\tau_{\Omega}-\tau\right)\right)\right] /\left(1+\omega^{2} \tau_{2}^{2}\right)
\end{aligned}
$$

Since $|X|$ and $|Y|$ depend on frequency and thereby can take values both smaller than and larger than one, we made the following classification:

$$
\begin{array}{ll}
|X| \gg 1 \text { and }|Y| \gg 1 & r=1 \text { and } s=Y /\left(X^{2}+Y^{2}\right), \\
|X| \gg|Y| \text { and }|X| \gg 1 & r=1 \text { and } s=Y \mid X^{2}, \\
|Y| \gg|X| \text { and }|Y| \gg 1 & r=1 \text { and } s=[1-\exp (-X) \cos Y] / Y, \\
|X| \ll 1 \text { and }|Y| \ll 1 & r=\frac{1}{2} X \text { and } s=\frac{1}{2} Y .
\end{array}
$$

In our case of gold-doped silicon we have

$$
\tau_{\mathbf{2}} / \tau=\left(n_{\mathrm{t}}-n_{2}\right) n_{2} /\left(n_{1} n_{\mathbf{t}}\right) \approx\left(n_{\mathrm{t}}-n_{2}\right) / n_{1} \gg 1
$$

(see section 4.2) at the temperatures under consideration. Taking into account the resistivity data and the applied d.c. bias voltages (at least $5 \mathrm{~V}$, then $\left.t_{n}=L^{2} /\left(\mu V_{0}\right) \leq 1.5 \times 10^{-6} \mathrm{~s}\right)$, we can use the inequality $\tau_{2} \gg \tau, \tau_{\Omega}, t_{n}$. If the applied d.c. bias voltage is so large that $1 / \tau_{\Omega}+1 / \tau \ll 1 / t_{n} \ll \tau_{\Omega} /\left(\tau \tau_{\Omega}\right)$ will hold, we can distinguish six frequency ranges according to increasing frequencies with the help of the above mentioned classification of $X$ and $Y$ and the inequality $\tau_{2} \gg \tau, \tau_{\Omega}, t_{n}$ :

I) $\omega \tau \ll\left[\tau t_{n} /\left(\tau_{2} \tau_{\Omega}\right)\right]^{2}$, consequently $|X| \gg 1$ :

$$
R_{\mathrm{p}}=R_{0}, \quad C_{\mathrm{p}}=C_{0}\left(\tau / t_{n}\right) .
$$


II) $\left[\tau t_{n} /\left(\tau_{2} \tau_{\Omega}\right)\right]^{\frac{1}{2}} \leq \omega \tau \ll \min \left\{\left\{\tau^{2} /\left[\tau_{2}\left(\tau+\tau_{\Omega}\right)\right]\right\}^{1}, t_{n /} \tau_{\Omega}\right\}$, so $|X| \leq 1$ and $|Y| \gg 1$ :

$$
R_{\mathbf{p}}=R_{0}, \quad C_{\mathbf{p}}=C_{0}\left\{1+\tau / t_{n}\left\{1-\exp \left[\cdots t_{n} /\left(\omega^{2} \tau_{2} \tau \tau_{\Omega}\right)\right] \cos \left[t_{n} /\left(\omega \tau \tau_{\Omega}\right)\right]\right\} .\right.
$$

In this frequency range we obtain for the local maxima and minima of $C_{\mathrm{p}}$ as a function of frequency: $C_{\max } \approx C_{0}\left(2 \tau / t_{n}\right)$ and $C_{\min } \approx C_{0} /\left(\omega_{k}^{2} \tau_{2} \tau_{\Omega}\right)$, respectively, where $\omega_{k} \approx t_{n} /\left(\tau \tau_{\Omega} k 2 \pi\right)$ and $k=1,2, \ldots$ In our cases

$$
\left\{\tau^{2} /\left[\tau_{2}\left(\tau+\tau_{\Omega}\right)\right]\right\}^{\frac{1}{2}}<t_{n} / \tau_{\Omega}
$$

always holds.

III) $\left\{\tau^{2} /\left[\tau_{2}\left(\tau+\tau_{\Omega}\right)\right]\right\}^{ \pm} \ll \omega \tau \ll t_{n} / \tau_{\Omega}$, therefore $|X| \ll 1$ and $|Y| \gg 1$ :

$$
\begin{aligned}
& R_{\mathrm{p}}=R_{0}, \\
& C_{\mathrm{p}}=C_{0}\left\{1+\tau / t_{n}\left\{1-\exp \left[-t_{n}\left(1 / \tau+1 / \tau_{\Omega}\right)\right] \cos \left[t_{n} /(\omega \tau \tau \Omega)\right]\right\}\right\} .
\end{aligned}
$$

Here we find for the local maxima and minima of $C_{\mathrm{p}}$ as a function of frequency:

$$
C_{\max } \approx C_{0}\left(2 \tau / t_{n}\right) \quad \text { and } \quad C_{\min } \approx C_{0}\left(2+\tau / \tau_{\Omega}\right) .
$$

IV) $\max \left\{t_{n} / \tau_{\Omega},\left\{\tau^{2} /\left[\tau_{2}\left(\tau+\tau_{\Omega}\right)\right]\right\}^{\frac{1}{2}}\right\} \ll \omega \tau \ll\left(\tau / \tau_{\Omega}\right)^{\frac{1}{2}}$, then $|X| \ll 1$ and $|Y| \ll 1$ :

$$
R_{\mathrm{p}}=R_{0}\left(t_{n} / 2 \tau_{\Omega}\right) /\left(\omega^{2} \tau^{2}\right), \quad C_{\mathbf{p}}=C_{0}\left(2 \tau / t_{n}\right) .
$$

If $\omega \tau=\left(\tau / \tau_{\Omega}\right)^{\frac{1}{2}}$ then $|X| \ll 1$ and $Y=0$, so that

$$
\begin{aligned}
& R_{\mathrm{p}}=R_{0}\left(t_{n} / 2 \tau_{\Omega}\right)\left[\left(\tau+\tau_{\Omega}\right) / \tau\right], \\
& C_{\mathrm{p}}=C_{0}\left(2 \tau / t_{n}\right)\left[\tau_{\Omega} /\left(\tau+\tau_{\Omega}\right)\right] \quad \text { and } \quad R_{\mathrm{p}} C_{\mathrm{p}}=R_{0} C_{0}=\tau_{\Omega} .
\end{aligned}
$$

V) $\left(\tau / \tau_{\Omega}\right)^{\frac{1}{2}} \ll \omega \tau \ll \tau / t_{n}$, thence $|X| \ll 1$ and $|Y| \ll 1$ :

$$
R_{\mathrm{p}}=R_{0}\left(t_{n} / 2 \tau_{\Omega}\right), \quad C_{\mathrm{p}}=C_{0}\left(2 \tau / t_{n}\right) /\left(\omega^{2} \tau^{2}\right) .
$$

VI) $\omega \tau \gg \tau \mid t_{n}$, so $|X| \ll 1$ and $|Y| \gg 1$ :

$$
R_{\mathfrak{p}} \approx R_{0}\left(t_{n} / \tau_{\Omega}\right) /\left[\left(t_{n} / \tau_{\Omega}\right)+1-\cos \omega t_{n}\right], \quad C_{\mathbf{p}}=C_{0} .
$$

The inequality $1 / t_{n}>1 / \tau_{\Omega}+1 / \tau$ prevails, provided that the d.c. bias voltage is larger than: $10,15,30,70$ and $140 \mathrm{~V}$, when the d.c. resistance $R_{0}$ is: $1.800,950,350,110$ and $50 \mathrm{k} \Omega$, respectively (see figs. 2,3 and 4). Here the symbols $\ll$ and $\gg$ mean smaller than or larger than a factor two or three.

Comparing the theoretical frequency ranges with our experimental results we may conclude that with increasing frequency (see figs. 2 and 4):

Range I can not be observed, owing to the limited sensitivity of the phase detector. Phase shifts less than $1^{\circ}$ are hardly measurable, therefore 
the frequency should be larger than about $3 \times 10^{3} /\left(R_{\mathrm{p}} C_{\mathrm{p}}\right)$ so that, if $R_{\mathrm{p}}=1 \mathrm{M} \Omega$ and $C_{\mathrm{p}}=10 \mathrm{pF}$ then $f>300 \mathrm{~Hz}$.

Ranges II and III were observed, where the capacitance shows a sinusoidal behaviour as function of the frequency. In fig. 4 we see that the value of $C_{\text {min }}$ increases with decreasing frequency, which means that we change from range III to range II. For instance, we can calculate $\tau_{2}$ from $C_{\min }$ at a d.c. bias voltage of $240 \mathrm{~V}$ where range II holds, hence we find:

$$
\tau_{2}=\left[\left(C_{\min } / C_{0}\right) \omega_{1}^{2} \tau_{\Omega}\right]^{-1} \approx 5 \times 10^{-3} \mathrm{~s},
$$

consequently

$$
n_{\mathrm{t}}-n_{2} \approx\left(\tau_{2} / \tau\right) n_{1} \approx 3 \times 10^{13} \mathrm{~cm}^{-3},
$$

which is in agreement with the gold dope and the degree of compensation of the gold-acceptor level (see also section 4.2). On the other hand, we find for this instance:

$$
\begin{aligned}
& {\left[\tau t_{n} /\left(\tau_{2} \tau_{\Omega}\right)\right]^{\frac{1}{2}}=2 \times 10^{-3}, \quad \omega_{1} \tau=3.6 \times 10^{-3},} \\
& \left\{\tau^{2} /\left[\tau_{2}\left(\tau+\tau_{\Omega}\right)\right]\right\}^{\frac{1}{2}}=8 \times 10^{-3} \quad \text { and } \quad \iota_{n} / \tau_{\Omega}=2.7 \times 10^{-2},
\end{aligned}
$$

so that the conditions for range II are fairly satisfied. The relaxation time $\tau$ can be calculated from the frequencies where $C_{p}$ reaches its local minima. For this minima obtains both in ranges II and III $: t_{n} /\left(\omega_{k} \tau \tau_{\Omega}\right)=k 2 \pi$, where $k=1,2, \ldots$. The $\tau$ values calculated from the high frequency minima, shown in fig. 2, are plotted in fig. 1.

Range IV is observed where $C_{p}$ is independent of the frequency, whereas $R_{p}$ rapidly decreases with increasing frequency. From the values obtained for $C_{\mathrm{p}}$ in this case, the relaxation time $\tau$ can be determined, for instance, if $R_{0}=950 \mathrm{k} \Omega$ and $V_{0}=100 \mathrm{~V}$ we find $\tau=\frac{1}{2}\left(C_{\mathrm{p}} / C_{0}\right) t_{n} \approx 6 \times 10^{-7} \mathrm{~s}$ which is in agreement with the values found from $g-r$ noise (see fig. 1 ).

In range $\mathrm{V} C_{\mathbf{p}}$ decreases monotonically with increasing frequency and $R_{\mathbf{p}}$ becomes independent of the frequency. But for a factor two, good agrcement exists between expcriment and theory with respect to the magnitude of $R_{p}$ in this case.

Range VI will be observed at frequencies greater than $2 \mathrm{MHz}$, since at $100 \mathrm{~V}$ d.c. bias voltage the transit time $t_{n}$ amounts to $7.7 \times 10^{-8} \mathrm{~s}$, hence $f \gg 1 /\left(2 \pi t_{n}\right) \approx 2 \times 10^{6} \mathrm{~Hz}$. Here $C_{\mathrm{p}}=C_{0}$, independent of frequency.

Therefore the theoretical behaviour as predicted in five different special ranges can be recognized in fig. 2 and fig. 4 going from lower to higher frequencies.

Fig. 3 shows the dependence of $C_{\mathbf{p}}$ on the applied d.c. bias voltage at four fixed frequencies. For d.c. bias voltages larger than about $30 \mathrm{~V}$, we shall compare these results of $C_{p}$ with the ranges I-VI. We have

$$
\begin{array}{crc}
\tau=8 \times 10^{-7} \mathrm{~s} & (\text { fig. 1), } & \tau_{\Omega}=1.2 \times 10^{-6} \mathrm{~s}, \\
\tau_{2}=5 \times 10^{-3} \mathrm{~s} & \text { and } & 3.3 \times 10^{-8} \leq t_{n} \leq 2.6 \times 10^{-7} \mathrm{~s} .
\end{array}
$$


Therefore at $1 \mathrm{kHz}$ we find:

$$
\omega \tau=5 \times 10^{-3}, \quad t_{n} / \tau_{\Omega} \geq 2.7 \times 10^{-2} \quad \text { and } \quad\left[\tau t_{n} /\left(\tau_{2} \tau_{\Omega}\right)\right]^{\frac{1}{2}} \leq 6 \times 10^{-3},
$$

consequently ranges II and III hold and $C_{\mathrm{p}}$ varies sinusoidally with $V_{\mathbf{0}}$. At $4 \mathrm{kHz}$ we have $\omega \tau=2 \times 10^{-2}$ and $2.7 \times 10^{-2} \leq t_{n} / \tau_{\Omega} \leq 2.2 \times 10^{-1}$, hence it appears that $\omega \tau$ becomes nearly equal to $t_{n} / \tau_{\Omega}$ at higher d.c. bias voltage, so that we arrive between range III and range IV; here the sinusoidal behaviour vanishes. At $100 \mathrm{kHz}$ we find $\omega \tau=0.5$ and $\left(\tau / \tau_{\Omega}\right)^{\frac{1}{2}}=0.8$ and consequently range IV holds. At $1.6 \mathrm{MHz}$ we have $\omega t_{n} \geq 0.8$ so that we are between range $\mathrm{V}$ and range $\mathrm{VI}$ and also in range VI, hence $C_{\mathrm{p}} \approx C_{0}$.

Fig. 3 shows also that $R_{\mathrm{p}}$ is inversely proportional to the applied d.c. bias voltage $V_{0}$ for frequencies of $100 \mathrm{kHz}$ and $1.6 \mathrm{MHz}$ and d.c. voltages larger than about $30 \mathrm{~V}$, i.e., $R_{\mathrm{p}}$ is proportional to the transit time $t_{n}$ (cf. ranges $I V-V)$. At lower frequencies (i.e. $1 \mathrm{kHz}$ and $4 \mathrm{kHz}$ ) the a.c. resistance increases slightly with increasing d.c. bias voltage. If the d.c. bias voltage ranges between $30 \mathrm{~V}$ and $240 \mathrm{~V}$, we find that the value of $t_{n} /\left(\omega \tau \tau_{\Omega}\right)$ varies between 43 and 5.3 at $1 \mathrm{kHz}$ and between 11 and 1.3 at $4 \mathrm{kHz}$, respectively. Therefore at d.c. bias voltages between $30 \mathrm{~V}$ and $240 \mathrm{~V}$ and at $1 \mathrm{kHz}$ ranges II or III are obtained and $R_{\mathrm{p}}=R_{0}$, hence the increase in $R_{\mathrm{n}}$ with increasing $V_{0}$ will be due to a decrease in the drift mobility $\left.{ }^{14}\right)$. At $4 \mathrm{kHz}$ we are between range III and range IV if $V_{0} \geq 100 \mathrm{~V}\left(i . e . t_{n}\right)$ $\left.\left(\omega \tau \tau_{\Omega}\right)<3\right)$. With the help of the expressions for $X, Y, r$ and $s$ we find, after some algebra, that if $t_{n} /\left(\omega \tau \tau_{\Omega}\right)$ is $\frac{3}{2} \pi, \frac{5}{4} \pi, \pi, \frac{3}{4} \pi, \frac{1}{2} \pi$ and $\frac{1}{4} \pi$, then $R_{\mathrm{p}}$ is equal to $1.0 R_{0}, 1.3 R_{0}, 1.4 R_{0}, 1.4 R_{0}, 1.7 R_{0}$ and $1.3 R_{0}$, respectively. Hence the increase in $R_{\mathrm{p}}$ with increasing $V_{0}$ up to $200 \mathrm{~V}$ will be due to both spacecharge injection and field-dependent mobility. The decrease in $R_{\mathrm{p}}$ at higher $V_{0}$ (from $200 \mathrm{~V}$ ) is a result from the transition to range IV.

The typical behaviour of the impedance for the eic-sample was due to the fact that the density of free electrons $n_{1}(x)$ depends on the applied electric field $E(x)$. In terms of eq. (4b) we observe that the current variation $\Delta J$, but for Langevin functions, is due to: (i) $q \mu n_{10} \Delta E$ associated with the normal ohmic conductance $1 / R_{0}$, (ii) $\varepsilon_{\mathrm{r}} \varepsilon_{\mathbf{0}} \partial \Delta E / \partial t$ which is connected with the normal geometric capacitance $C_{0}$, and (iii) $q \mu E_{0} \Delta n_{1}$ which is responsible for the deviating behaviour of the impedance when compared to a simple $\mathrm{RC}$ circuit.

4.4. Noise of the eic-sample. With respect to the short-circuit current fluctuations given by eq. (11), we may distinguish three limiting theoretical cases:

A) $|a L| \ll 1$. This case is obtained if both $1 / t_{n} \gg 1 / \tau+1 / \tau_{\Omega}$ and $t_{n} / \tau_{\Omega} \ll \omega \tau \ll \tau / t_{n}$, then $f(a L) /|g(a L)|^{2}=\frac{4}{3}$.

B) $\operatorname{Re}(a L) \ll 1,|\operatorname{Im}(a L)| \gg 1$. This requires $1 / t_{n} \gg 1 / \tau+1 / \tau_{\Omega}$ and at the same time $\omega t_{n} \gg 1$ or $\left[\tau t_{n /}\left(\tau_{2} \tau_{\Omega}\right)\right]^{1} \ll \omega \tau \ll t_{n} / \tau_{\Omega}$; then $f(a L) /|g(a L)|^{2}=2$. 
C) $\operatorname{Re}(a L) \gg 1$. This implies either $1 / t_{n} \gg 1 / \tau+1 / \tau_{\Omega}$ and

$$
\omega \tau \ll\left[\tau t_{n} /\left(\tau_{2} \tau_{\Omega}\right)\right]^{\mathrm{t}} \quad \text { or } \quad 1 / t_{n} \ll 1 / \tau+1 / \tau_{\Omega}
$$

(i.e. low-injection level); then $f(a L) /|g(a L)|^{2}=1$.

If $1 / t_{n} \gg 1 / \tau+1 / \tau_{\Omega}$, we find that the g-r noise largely exceeds the Nyquist noise in the frequency range considered (by a factor larger than $10^{2}$ at angular frequencies $\left.\omega \leq 1 / \tau\right)$, hence the correction of the spectra for Nyquist noise (dependent on $V_{0}$ !) will be unambiguous [cf. eq. (11)].

Comparing these limiting theoretical cases with our experimental data, given in fig. 5 , we have for the spectrum, where $I=9 \mu \mathrm{A}, 1 / t_{n}=9 \times$ $\times 10^{5} \mathrm{~s}^{-1}$ and $1 / \tau+1 / \tau_{\Omega}=2.6 \times 10^{6} \mathrm{~s}^{-1}$ and consequently case $\mathrm{C}$ holds. This spectrum shows the ideal frequency behaviour, namely $\sim 1$ / $\left(1+\omega^{2} \tau^{2}\right)$. In this case we find $N_{1}=6 \times 10^{7}$ and hence it appears that $\mu=L^{2} /\left(q N_{1} R_{0}\right)=1.4 \times 10^{3} \mathrm{~cm}^{2} / \mathrm{Vs}$ which is in close agreement with the literature $\left.{ }^{13}\right)$. With respect to the spectrum, where $I=148 \mu \mathrm{A}$, we find $1 / t_{n}=1.4 \times 10^{7} \mathrm{~s}^{-1}$ and so $1 / t_{n} \gg 1 / \tau+1 / \tau_{\Omega}$. In this case we obtain aftcr some algebra: (i) case $\mathrm{A}$ holds between $20 \mathrm{kHz}$ and $2 \mathrm{MHz}$, (ii) case $\mathrm{B}$ holds between $900 \mathrm{~Hz}$ and $20 \mathrm{kHz}$ and also for frequencies higher than $2 \mathrm{MHz}$, (iii) case $\mathrm{C}$ holds at frequencies below $900 \mathrm{~Hz}$. Although the agreement between theory and experiment is not perfect we see that the deviation from the ideal shape of the g-r spectrum becomes clear at higher d.c. currents. Moreover, we see in fig. 5 that at frequencies below about $20 \mathrm{kHz}$ the plateau level of the g-r noise at high currents is larger than the low current plateau level (in accordance with case B). However, in contrast with case $\mathrm{A}$ the $\mathrm{g}-\mathrm{r}$ noise density becomes smaller than the expected density at frequencies higher than $50 \mathrm{kHz}$.

In view of the limiting cases $\mathrm{A}, \mathrm{B}$ and $\mathrm{C}$ the spectral density of the Nyquist current-noise represented in eq. (11) varies between $4 k T / R_{0}$ and $8 k T / R_{0}$. On the other hand, the Nyquist current noise in thermodynamic equilibrium is $4 k T / R_{\mathrm{p}}(\omega)$, where $R_{\mathrm{p}}(\omega)$ is the equivalent parallel resistance. However, in our case the eic-sample under d.c. bias is out of thermodynamic equilibrium so that the Nyquist current noise will not be equal to $4 k T / R_{\mathrm{p}}(\omega)$. Note that $R_{\mathrm{p}}(\omega)$ may deviate strongly from $R_{\mathbf{0}}$ (see section 4.3).

In our samples the Nyquist current noise under d.c. bias voltages can be studied only at very high frequencies, since at frequencies below $1 \mathrm{MHz}$ the Nyquist current noise is masked completely by g-r noise.

5. Conclusions. Although in the calculations concerning the impedance, Nyquist noise and g-r noise of SCL diodes: (i) the diffusion terms have been neglected (ii) the approximation is made that the densities of free electrons and empty traps are not affected in the larger part of the samples at the injection levels under consideration and consequently Ohm's law 
holds and (iii) the boundary condition $e(\omega, 0)=0$ is used in order to describe the properties of an injecting contact, a remarkably good description of the behaviour of the diode is obtained.

We may thus conclude that the typical behaviour of the a.c. smallsignal impedance and the $g-r$ noise of our single-injection solid-state diodes can be satisfactorily explained by our extension of the theory as developed by Zijlstra and Driedonks ${ }^{1,2}$ ) in the ohmic regime.

Acknowledgements. The author is indebted to Professor C. Th. J. Alkemade, Dr. F. Driedonks and Dr. R. J. J. Zijlstra for valuable discussions and for critically reading the manuscript. He is grateful to Drs S. Kruizinga and Mr. F. Wollenberg for their technical advice during this work. This work was performed as part of the research program of the "Stichting voor Fundamenteel Onderzoek der Materie" (F.O.M.) with financial support from the "Nederlandse Organisatie voor Zuiver-Wetenschappelijk Onderzoek" (Z.W.O.).

\section{REFERENCES}

1) Zijlstra, R. J. J. and Driedonks, F., Physica 50 (1970) 331.

2) Driedonks, F., Ph. D. Thesis, University of Utrecht (1970).

3) Zijlstra, R. J. J., Solid-State Electronics 14 (1971) 365.

4) Lampert, M. A. and Mark, P., Current Injection in Solids, Academic Press (New York, 1970).

5) Shockley, W. and Prim, R. C., Phys. Rev. 90 (1953) 753.

6) Bullis, W. M., Solid-State Electronics 9 (1966) 143.

7) Colligan, M. B. and Van Vliet, K. M., Phys. Rev. 171 (1968) 881.

8) Van der Ziel, A., Solid-State Electronics 9 (1966) 1139.

9) Zijistra, R. J. J., private communication.

10) Sullivan, M. V. and Eigler, J. A., J. Electrochem. Soc. 104 (1957) 226.

11) Boltaks, B. I., Diffusion in Semiconductors, Infosearch Limited (London, 1963).

12) Wilcox, W. R., La Chapelle, T. J. and Forbes, D. H., J. Electrochem. Soc. 111 (1964) 1377.

13) Smith, R. A., Semiconductors, University Press (Cambridge, 1961).

14) Ryder, E. J., Phys. Rev. 90 (1953) 766.

15) Lax, M., Phys. Rev. 119 (1960) 1502. 